\title{
Studying Li Dynamics in a Gas-Phase Synthesized Amorphous Oxide by NMR and Impedance Spectroscopy
}

\author{
By Viktor Epp ${ }^{1,3, *}$, Christian Brünig ${ }^{2}$, Michael Binnewies ${ }^{2}$, Paul Heitjans ${ }^{3}$, and \\ Martin Wilkening ${ }^{1,3}$ \\ ${ }^{1}$ Graz University of Technology, Institute for Chemistry and Technology of Materials, Stremayrgasse 9, \\ A-8010 Graz, Austria \\ 2 Leibniz University Hannover, Institute of Inorganic Chemistry, Callinstr. 9, D-30167 Hannover, \\ Germany \\ ${ }^{3}$ Leibniz University Hannover, Institute of Physical Chemistry and Electrochemistry, Callinstr. 3-3A, \\ D-30167 Hannover, Germany
}

(Received February 9, 2012; accepted in revised form April 16, 2012)

(Published online May 14, 2012)

\section{Li Diffusion / Solid State NMR / Impedance Spectroscopy / Non-Stoichiometric Compounds}

Li diffusion parameters of a structurally disordered $\mathrm{Li}$-Al-Si-oxide prepared by gas-phase synthesis were complementarily investigated by both time-domain NMR techniques and impedance spectroscopy. The first include ${ }^{7} \mathrm{Li}$ NMR spin-lattice relaxation (SLR) measurements in the laboratory as well as in the rotating frame of reference. An analysis of variable-temperature NMR line widths point to an activation energy $E_{\mathrm{a}}$ of approximately $0.6 \mathrm{eV}$. The value is confirmed by rotating-frame SLR NMR data recorded at approximately $11 \mathrm{kHz}$. Above room temperature the low-temperature flank of a diffusion-induced rate peak shows up which can be approximated by an Arrhenius law yielding $E_{\mathrm{a}}=0.56(1) \mathrm{eV}$. This is in very good agreement with the result obtained from ${ }^{7} \mathrm{Li}$ spin-alignment echo (SAE) NMR being sensitive to even slower Li dynamics. For comparison, dc-conductivity measurements, probing long-range motions, yield $E_{\mathrm{a}}=0.8 \mathrm{eV}$. Interestingly, lowtemperature SAE NMR decay rates point to localized Li motions being characterized with a very small activation energy of only $0.09 \mathrm{eV}$.

\section{Introduction}

The precise measurement of diffusion parameters in solids is a vital topic in materials science [1-4]. In particular, the investigation of Li dynamics over a wide length scale turns out to be highly important to understand the underlying structure-property relationships governing ion transport in materials being relevant for battery applications [515]. For instance, fast ion conductors are needed to develop powerful all-solid-state

\footnotetext{
* Corresponding author. E-mail: epp@pci.uni-hannover.de
} 
lithium-ion batteries which are regarded to play a key role in the development of new energy storage systems [16-18].

In many cases the introduction of structural disorder leads to an improvement of the diffusion parameters. For example, $\mathrm{Li}$ diffusion in nanocrystalline $\mathrm{LiNbO}_{3}$ (and $\mathrm{LiTaO}_{3}$ ), [19-21] which was prepared by high-energy ball milling, is enhanced by several orders of magnitude compared to its structurally ordered crystalline counterpart. Especially in the case of amorphous materials, offering both local movement modes and long-range migration pathways, diffusion parameters such as jump rates and activation energies might differ from each other when probed on different length scales and time scales, respectively [20,22-24]. In order to draw a complete picture of the different motions taking place in structurally disordered materials, dynamic parameters should preferably be probed by complementary techniques being sensitive to $\mathrm{Li}$ dynamics occurring on different time scales $[10,13,25,26]$. Here, exemplarily a gasphase synthesized (metastable) non-stoichiometric amorphous oxide composed of $\mathrm{Li}$, $\mathrm{Si}$ and $\mathrm{Al}$ served as a highly disordered model substance which was comprehensively investigated by both variable-temperature NMR and impedance spectroscopy.

The present study aims at the comparison of diffusion and transport parameters, respectively, obtained from quite different methods which are sensitive to short-range and long-range Li motions. In particular, besides NMR line shape measurements, variabletemperature ${ }^{7} \mathrm{Li}$ NMR spin-lattice relaxation (SLR) rates in both the laboratory and rotating frame of reference [24] have been measured. Whereas the first are sensitive to very fast Li motions with jump rates in the order of the Larmor frequency used, which is in the $\mathrm{MHz}$ range here, SLR rates probed in the rotating frame of reference are usually sampled at locking frequencies in the order of some kHz. Thus, the latter measurements are per se sensitive to much slower Li dynamic processes with jump rates in the order of $10^{4} \mathrm{~s}^{-1}$. Even slower Li jump processes were probed by the so-called NMR spinalignment echo (SAE) technique [22,23,27-32]. ${ }^{7} \mathrm{Li}$ SAE NMR two-time correlation functions were recorded at different temperatures by the use of the Jeener-Broekaert pulse sequence. In this way, the method is sensitive to Li jump rates with values down to $10^{1} \mathrm{~s}^{-1}[7,28]$. Finally, impedance spectroscopy measurements covering a frequency range from $10 \mathrm{~Hz}$ to $1 \mathrm{MHz}$ complement the study.

\section{Experimental}

The non-equilibrium oxide studied was synthesized via a gas-phase reaction in a threezone tube furnace resulting in a metastable, highly non-stoichiometric powder containing $\mathrm{Li}, \mathrm{Al}, \mathrm{Si}$, and $\mathrm{O}$. The precursors used for the studied sample were pure lithium chloride (Sigma-Aldrich, > 99\%), aluminum chloride (Sigma-Aldrich, 98\%), and silicon tetrachloride (Riedel-de-Haen, $>99 \%$ ). A detailed description of the procedure including the setup of the apparatus used for the gas-phase synthesis employed can be found in Refs. [33,34]. Let us remark that the synthesis route chosen has the great advantage of providing a very easy way to adjust the sample stoichiometry. Up to now, we were able to synthesize a series of non-stoichiometric samples with quite different compositions. These are included in Fig. 1a; here, exemplarily the sample numbered "57" (marked with a cross) was studied. For comparison, the stoichiometric alumosilicates 


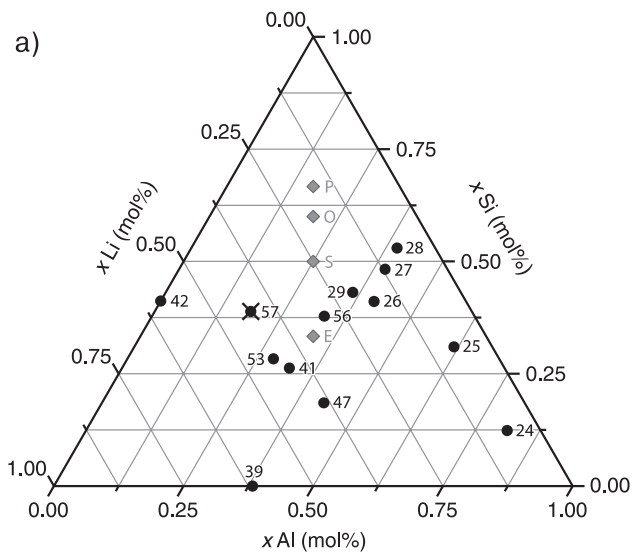

b)

Fig. 1. (a) Li-Al-Si-O-phase diagram; so far, a series of non-stoichiometric samples, labelled with numbers, have been prepared in our laboratory. $\mathrm{P}, \mathrm{O}, \mathrm{S}$, and $\mathrm{E}$ highlight the compounds petalite, lithium orthoclase, spodumene and eucryptite. (b) SEM image of sample " 57 ", see (a).

petalite $\mathrm{LiAlSi}_{4} \mathrm{O}_{10}(\mathrm{P})$, lithium orthoclase $\mathrm{LiAlSi}_{3} \mathrm{O}_{8}(\mathrm{O})$, spodumene $\mathrm{LiAlSi}_{2} \mathrm{O}_{6}(\mathrm{~S})$ and eucryptite $\mathrm{LiAlSiO}_{4}(\mathrm{E})$ are also included. Sample "57" is characterized by an $\mathrm{Al}: \mathrm{Li}: \mathrm{Si}$ ratio of approximately $1: 2.3: 2.1$ (see Fig. 1a). This ratio was determined by inductively coupled plasma atomic emission spectroscopy (ICP-AES). The sample was found to be X-ray amorphous; scanning electron microscopy (SEM) images show that it consists of globular particles with diameters ranging from $200 \mathrm{~nm}$ to $750 \mathrm{~nm}$ (see Fig. 1b).

Solid-state ${ }^{7} \mathrm{Li}$ NMR measurements, carried out to get insights into the Li dynamics of the amorphous oxide, were performed by using a modified Bruker MSL-100 equipped with a Kalmus $400 \mathrm{~W}$ amplifier. The spectrometer is connected to a fieldvariable Oxford cryomagnet which is operated at a (nominal) resonance frequency $\omega_{0} / 2 \pi$ of $77.7 \mathrm{MHz}$. A standard broadband probe (Bruker) was used to sample NMR spectra and spin-lattice relaxation rates. The temperature in the direct neighbourhood of the sample was monitored with an ITC4 temperature controller (Oxford) using a Ni-CrNi thermocouple. All measurements were performed at temperatures ranging from $173 \mathrm{~K}$ to $453 \mathrm{~K}$. The temperature was either adjusted by a stream of heated air $(T>300 \mathrm{~K})$ or by freshly evaporated nitrogen $(T<300 \mathrm{~K})$. Prior to the NMR measurements the sample was extensively vacuum dried and then fire-sealed in a glass tube ( $4 \mathrm{~mm}$ in diameter and $4 \mathrm{~cm}$ in length).

${ }^{7} \mathrm{Li}$ NMR spin-lattice relaxation rates $1 / T_{1}$ were acquired with the saturation recovery pulse sequence: $10 \times \pi / 2-t_{\mathrm{i}}-\pi / 2[24,35]$. The $\pi / 2$ pulse length was approximately $7 \mu \mathrm{s}$; the first 10 pulses, separated by a delay time of $50 \mu \mathrm{s}$, are used to destroy longitudinal spin magnetization $M_{z}(t)$. The subsequent recovery of $M_{z}(t)$ is recorded as a function of 14 different delay times $t_{\mathrm{i}}(i=1 \ldots 14)$. Similarly, ${ }^{7} \mathrm{Li}$ NMR relaxation rates $1 / T_{1 \rho}$ in the rotating frame of reference were measured. For this purpose the spin-lock technique [35-41] was used to record $M_{\rho}\left(t_{\text {lock }}\right)$ transients. The duration of the locking pulse $t_{\text {lock }}$ was varied from $1 \mathrm{~ms}$ up to $40 \mathrm{~s}$. The corresponding locking frequency $\omega_{1} / 2 \pi$ was estimated to be of the order of $v_{\text {lock }}=11 \mathrm{kHz}$. 
As already mentioned above, ${ }^{7} \mathrm{Li}$ NMR spin-alignment echoes were recorded using the pulse sequence introduced by Jeener and Broekaert $[42,43]: \pi / 2_{X}-t_{\mathrm{p}}-\pi / 4_{Y}-t_{\mathrm{m}}-$ $\pi / 4_{X}-t_{\mathrm{p}}$ - echo, where $X$ and $Y$ denote the phase shifts of the radio frequency (rf) pulses used. In the ideal case, the first two rf pulses, separated by the preparation time $t_{\mathrm{p}}$ which has to be chosen as short as possible [30,44-48] (here $t_{\mathrm{p}}=10 \mu \mathrm{s}$ was used), create (quadrupolar) spin-alignment order. Li motions affecting quadrupole spin-order take place during the mixing period determined by $t_{\mathrm{m}}\left(1 \mu \mathrm{s}<t_{\mathrm{m}}<100 \mathrm{~s}\right)$. The third or reading rf pulse of the Jeener-Broekaert pulse sequence transforms spin-alignment back into an observable transverse coherent magnetization giving rise to a stimulated echo at $t=t_{\mathrm{p}}$. Damping of the echo intensity $S_{2}$ with increasing mixing time leads to a two-time correlation function containing, in general, information on the dynamics $[49,50]$ as well as geometry [51] of the diffusion process studied. The recycle delay was set to at least $5 \times T_{1}$.

Impedance measurements were carried out at frequencies ranging from $5 \mathrm{~Hz}$ to $13 \mathrm{MHz}$ with a home-built impedance cell connected to an HP 4192A RF impedance analyzer. The cell was placed in a tube furnace controlled by an OMRON E5CK temperature unit. For the impedance measurements, the powder sample was pressed into a pellet without any binder by applying a uniaxial pressure of about $1 \mathrm{GPa}$. Pt electrodes were applied by using platinum powder of high purity (Merck, 99.9\%). The resulting pellet had a thickness of about $1 \mathrm{~mm}$ and a diameter of $8 \mathrm{~mm}$. In order to exclude the influence of water upon the measurements, the sample was heated above $400 \mathrm{~K}$ in a dry nitrogen atmosphere for several hours. Subsequently, the impedance spectra were recorded between room temperature and $670 \mathrm{~K}$ in the same atmosphere using a homebuilt impedance cell with a four-terminal configuration.

\subsection{Results and discussion}

\subsection{1 ${ }^{7} \mathrm{Li}$ NMR measurements}

In many cases, a ${ }^{7} \mathrm{Li}$ NMR line-shape analysis provides first insights into the short time-scale Li ion dynamics of a given (diamagnetic) Li ion conductor. In Fig. 2a four selected NMR spectra recorded under static conditions at the temperatures indicated are shown. In the so-called rigid lattice regime of the sample, i.e., here at temperatures below $320 \mathrm{~K}$, the ${ }^{7} \mathrm{Li}$ NMR spectra consist of a single dipolarly broadened, almost purely Gaussian shaped line. At this temperature Li diffusion is too low to affect the line width of the spectrum. This drastically changes when the Li jump rate reaches the order of the NMR line width. As a result of rapid Li exchange processes taking place at elevated temperatures, (homonuclear) dipole-dipole interactions are increasingly averaged leading to the typical motional narrowing of the line. Here, with rising temperature the shape of the NMR line gradually changes into a pure Lorentzian. At intermediate temperatures the NMR lines seem to show the characteristics of a two-component line shape indicating distinctly mobile Li ions. As expected for an amorphous solid this might point to a heterogeneous dynamics present being similar to that found in other structurally disordered, glassy materials [19,22].

Plotting the NMR line width, i.e., the full width at half maximum (FWHM), as a function of $T$, it can be seen that motional narrowing sets in at approximately 

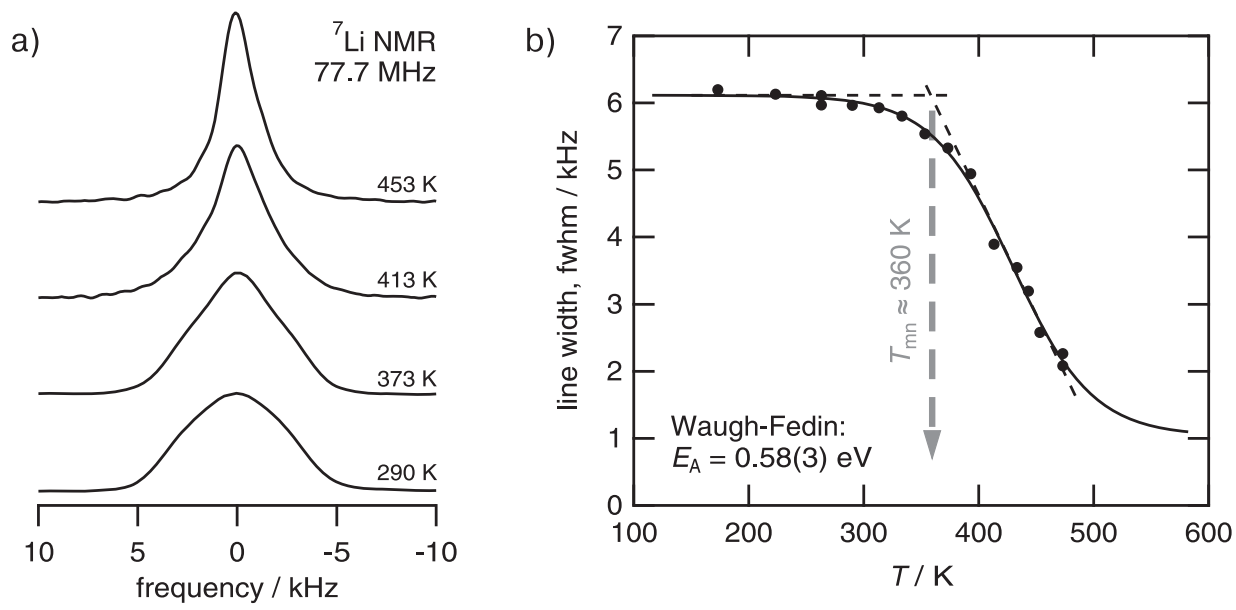

Fig. 2. (a) Selected ${ }^{7} \mathrm{Li}$ NMR spectra of the structurally disordered $\mathrm{Li}-\mathrm{Si}$-Al-oxide recorded at $77.7 \mathrm{MHz}$. (b) ${ }^{7} \mathrm{Li}$ NMR line width (full width at half maximum, fwhm) as a function of temperature. The onset of motional narrowing is observed at $T_{\mathrm{mn}} \approx 360 \mathrm{~K}$ and points to an activation energy of approximately $0.6 \mathrm{eV}$ when estimated using the relation introduced by Waugh and Fedin [52]. The solid line is drawn to guide the eye.

$T_{\mathrm{mn}}=360 \mathrm{~K}$ (see Fig. 2b). Since the rigid-lattice line width is in the order of some $\mathrm{kHz}$, this points to a mean $\mathrm{Li}$ jump rate of approximately $10^{3} \mathrm{~s}^{-1}$ at $T_{\mathrm{mn}}$. Using the expression introduced by Waugh and Fedin [52], an activation energy of about $0.58 \mathrm{eV}$ can be roughly estimated. The Waugh-Fedin expression relates the onset temperature of motional narrowing with the activation energy of the diffusion process and is given by: $E_{\mathrm{a}, \mathrm{mn}} / \mathrm{eV}=1.67 \times 10^{-3} \cdot T_{\mathrm{mn}} / \mathrm{K}$.

In contrast to line shape measurements, ${ }^{7} \mathrm{Li}$ NMR relaxometry provides a better quantitative access to Li diffusion parameters in solids. In the Arrhenius diagram of Fig. 3a ${ }^{7} \mathrm{Li}$ NMR SLR rates recorded in the laboratory $\left(1 / T_{1}\right)$ as well as in the rotating $\left(1 / T_{1 \rho}\right)$ frame of reference are shown as a function of inverse temperature. In each case the SLR NMR rate was obtained by fitting the corresponding magnetization transients with stretched exponentials. As an example, in Fig. $3 b$ typical (normalized) magnetization transients $M_{\rho}\left(t_{\text {lock }}\right)$ recorded at different temperatures are shown. The solid lines show fits with $M_{\rho}\left(t_{\text {lock }}\right) \propto \exp \left(-\left(t_{\text {lock }} / T_{1 \rho}\right)^{\gamma}\right)$. Interestingly, the stretching exponent $\gamma$ turned out to scatter around a mean value given by $\gamma_{\mathrm{m}}=0.5$. Hence, all fits were performed with a fixed stretching parameter $\gamma=\gamma_{\mathrm{m}}$. Most likely, $\gamma_{\mathrm{m}}=0.5$ points to localized relaxation centres such as paramagnetic impurities, see, e. g., Refs. [53,54].

Contrary to the $\operatorname{SLR}_{\rho}$ rates in the rotating frame of reference, the $1 / T_{1}$ rates measured at $77.7 \mathrm{MHz}$ are governed by non-diffusive background relaxation over almost the whole temperature range covered (see Fig. 3a). This manifests in a weak temperature dependence which can be described in terms of a power law $1 / T_{1, \text { nd }} \sim T^{\alpha}$ with $\alpha \approx 0.6$ (dotted lines in Fig. 3a). Most likely, it is, to a certain degree, caused by spin-diffusion (i.e., flip-flop processes) due to paramagnetic impurities which are also responsible for the stretching of the corresponding (non-exponential) transients. Furthermore, lattice vibrations or localized (i.e., non-translational) motions, for example, 
a)

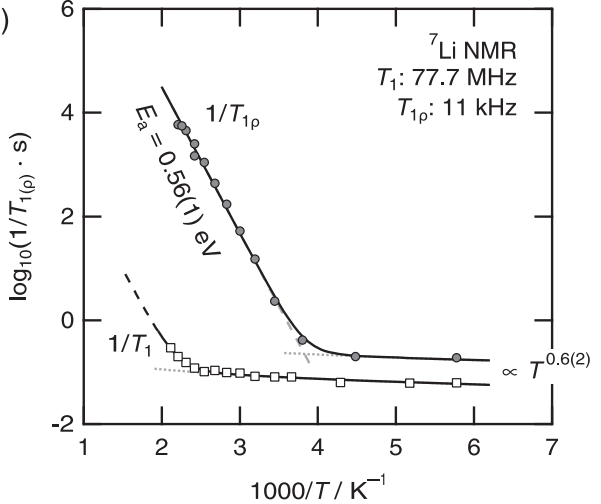

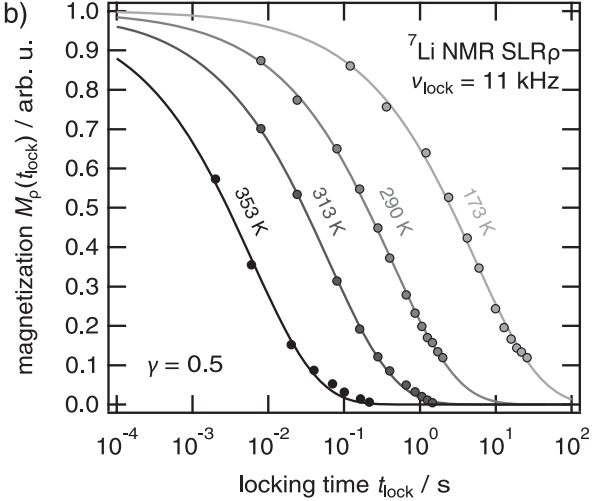

Fig. 3. (a) ${ }^{7} \mathrm{Li}$ NMR SLR rates in the laboratory $\left(1 / T_{1}, \square\right)$ and rotating $\left(1 / T_{1 \rho}, \bullet\right)$ frame of reference as a function of inverse temperature 1/T. Data have been recorded at a Larmor frequency of $77.7 \mathrm{MHz}$ and a locking frequency of $11 \mathrm{kHz}$, respectively. The corresponding transients $M_{\rho}\left(t_{\text {lock }}\right)$ are shown in (b). The rates reveal two regimes viz the diffusion-controlled flank of a $1 / T_{1(\rho)}(1 / T)$ rate peak and a nondiffusive background contribution showing up below $250 \mathrm{~K}$. The latter is characterized by a weakerthan-activated temperature dependence following a power law $1 / T_{1(\rho) \text {,nd }} \propto T^{0.6(2)}$ (grey dotted lines). The diffusion-induced low- $T$ flank exhibits Arrhenius behaviour $1 / T_{1(\rho) \text {,diff }} \propto \exp \left(-E_{\mathrm{a}} / k_{\mathrm{B}} T\right)$ and yields $E_{\mathrm{a}}=$ $0.56(1) \mathrm{eV}$ (see dashed line). (b) ${ }^{7} \mathrm{Li}$ NMR SLR $\mathrm{SL}_{\rho}$ magnetization transients $M_{\rho}\left(t_{\text {lock }}\right)$ for four selected temperatures plotted $v s$. duration of the locking pulse. The locking frequency $v_{\text {lock }}$ was set to $\omega_{1} / 2 \pi=11 \mathrm{kHz}$. The solid lines represent fits of stretched exponentials with a fixed stretching exponent $\gamma$. See text for further details.

might contribute to the low-temperature NMR SLR rates $1 / T_{1}$ and $1 / T_{1 \rho}$, respectively. Whereas for pure spin-diffusion, $1 / T_{1, \text { nd }}$ is expected to be independent of $T$, strictly localized motions, in the sense of being the analogy of the well-known nearly constant loss (NCL) phenomenon in dielectric spectroscopy [55-59], would lead to $1 / T_{1, \text { nd }} \sim T^{\alpha}$ with $\alpha \rightarrow 1$ [60-62]. For comparison, NMR SLR solely induced by lattice vibrations is expected to yield $1 / T_{1, \text { nd }} \sim T^{2}[63,64]$.

At temperatures above $410 \mathrm{~K}$ the SLR rates shown in Fig. 3a are increasingly influenced by translational Li ion diffusion processes. Therefore, the beginning of the low-temperature flank of the corresponding rate peak shows up. Provided the SLR NMR rates are induced by Li diffusion processes, reducing the Larmor frequency should shift this flank towards lower temperatures. According to the model introduced by Bloembergen, Purcell and Pound [65] the rate $1 / T_{1}$ on the low- $T$ flank, that is, in the limit $\omega_{0} \tau \gg 1$, is expected to show a quadratic frequency dependence.

The same dependency shows up when $\omega_{0}$ is replaced by $\omega_{1}, i$. e., when the resonance frequency in the $\mathrm{MHz}$ regime is replaced by a locking frequency being in the order of some $\mathrm{kHz}$ [24]. As can be clearly seen in Fig. 3a, at $T>265 \mathrm{~K}$ the corresponding NMR $\mathrm{SLR}_{\rho}$ rates probed in the rotating frame of reference exhibit a pronounced diffusioninduced low- $T$ flank following Arrhenius behaviour $1 / T_{1 \rho \text {, diff }} \propto \exp \left(-E_{\mathrm{a}} / k_{\mathrm{B}} T\right)$ with an activation energy of approximately $E_{\mathrm{a}}=0.56 \mathrm{eV}$ (see the dashed line in Fig. 3a). However, up to $T=450 \mathrm{~K}$ no ${ }^{7} \mathrm{Li} \mathrm{NMR} \mathrm{SLR}$ maximum shows up. This might emerge at temperatures higher than $500 \mathrm{~K}$. Below $250 \mathrm{~K}$ a non-diffusive background contribution is observed; the corresponding rates $1 / T_{1 \rho, \text { nd }}$ depend only weakly on temperature, which 
a)

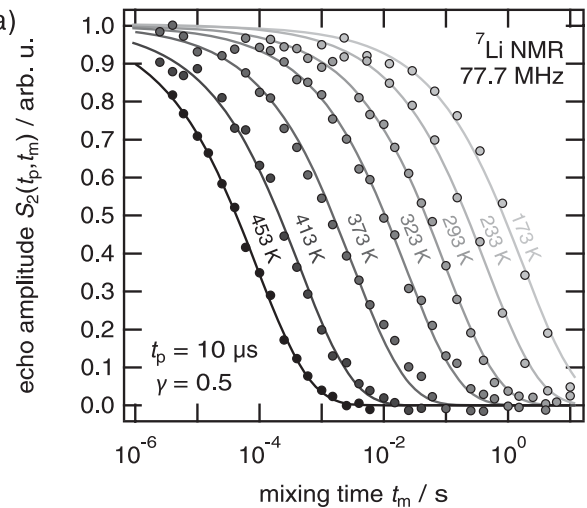

b)

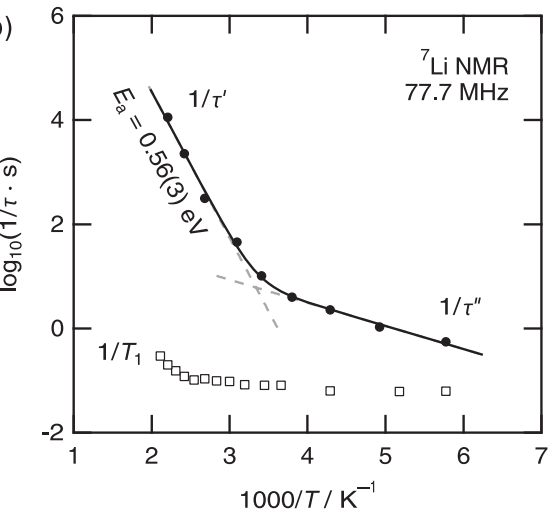

Fig. 4. (a) ${ }^{7} \mathrm{Li}$ NMR SAE decay curves $S_{2}\left(t_{\mathrm{p}}, t_{\mathrm{m}}\right)$ recorded at the temperatures indicated. $S_{2}\left(t_{\mathrm{p}}, t_{\mathrm{m}}\right)$ has been plotted in a semi-log representation as a function of $t_{\mathrm{m}}$. Data were measured at fixed preparation time, $t_{\mathrm{p}}=10 \mu \mathrm{s}$ and at $77.7 \mathrm{MHz}$. The solid lines are fits according to a stretched exponential using a fixed stretching exponent $\gamma=0.5$. (b) Corresponding ${ }^{7} \mathrm{Li} \mathrm{NMR} \mathrm{SAE} \mathrm{decay} \mathrm{rates}(\bullet)$ as a function of the inverse temperature. The rates are a sum of two contributions viz a weakly temperature-dependent one showing up at $T<280 \mathrm{~K}$ and a strongly thermally-activated one becoming apparent above $290 \mathrm{~K}$. The latter shows Arrhenius behaviour with $E_{\mathrm{a}}=0.56 \mathrm{eV}$. For comparison, the ${ }^{7} \mathrm{Li}$ NMR SLR rates $1 / T_{1}$ ( $\square$ ), see Fig. 3b, are also shown.

is practically the same behaviour as found for the SLR rates recorded in the laboratory frame of reference (see above).

Assuming that the parameters describing the temperature dependencies of the SLR rates found, viz $E_{\mathrm{a}}$ and $\alpha$, i.e., of both the diffusion-induced and the non-diffusive contribution, are the same for $1 / T_{1}$ and $1 / T_{1 \rho}$ a joint fit can be applied to the two data sets. The solid lines in Fig. 3a represent such a fit using a sum of the two contributions $1 / T_{1(\rho)}=1 / T_{1(\rho) \text {,diff }}+1 / T_{1(\rho) \text {,nd }}$, linking the parameters $E_{\mathrm{a}}$ and $\alpha$. Here, it turned out that $E_{\mathrm{a}}=0.57(1) \mathrm{eV}$ and $\alpha=0.6(2)$. However, since SLR NMR and $\mathrm{SLR}_{\rho}$ NMR probe diffusion parameters on different length scales, the slope of the low- $T$ flank of the SLR NMR rates, being sensitive to short-range Li motions, might be smaller than $E_{\text {a }}$ found for the corresponding flank of the $\mathrm{SLR}_{\rho} \mathrm{NMR}$ rate peak. Indeed, a dependence of $E_{\text {a }}$ on the time window used to study $\mathrm{Li}$ dynamics has also been found for spodumene $\left(\mathrm{LiAlSi}_{2} \mathrm{O}_{6}\right)$ studied by both NMR and ac impedance spectroscopy [66,67].

In Fig. 4a ${ }^{7}$ Li SAE NMR (normalized) decay curves $S_{2}\left(t_{\mathrm{p}}, t_{\mathrm{m}}\right)$ are presented which have been recorded at fixed preparation time, $t_{\mathrm{p}}=10 \mu \mathrm{s}$. Normalization means that the curves were scaled such that echo intensities range between 0 and 1 . The echo amplitudes $S_{2}\left(t_{\mathrm{p}}, t_{\mathrm{m}}\right)$ recorded at different temperatures can be well described by stretched exponentials according to

$$
S_{2}\left(t_{\mathrm{p}}, t_{\mathrm{m}}\right) \propto \exp \left(-\left(t_{\mathrm{m}} / \tau\right)^{\gamma}\right) \quad \text { with } \quad \tau=f(T) .
$$

As in the case of the NMR SLR ${ }_{\rho}$ measurements, the (fractal) stretching exponent turned out to scatter around a mean value $\gamma_{\mathrm{m}}=0.5$. Consequently, we used a fixed (temperature independent) stretching exponent $\left(\gamma=\gamma_{\mathrm{m}}\right.$, see solid lines in Fig. 4a) to extract the echo decay rates $1 / \tau(T)$. These are shown in Fig. $4 \mathrm{~b}$ using an Arrhenius representation. 
As can be clearly seen, the overall rate $1 / \tau$ is composed of two contributions and can be well described as a sum of the individual rates $1 / \tau^{\prime}$ and $1 / \tau^{\prime \prime}$

$$
1 / \tau=1 / \tau^{\prime}+1 / \tau^{\prime \prime}
$$

$1 / \tau^{\prime}$ as well as $1 / \tau^{\prime \prime}$ follow Arrhenius behaviour $1 / \tau^{i} \propto \exp \left(-E_{\mathrm{a}}^{i} / k_{\mathrm{B}} T\right)$. While $1 / \tau^{\prime}$ shows a strong temperature dependence characterized by $E_{\mathrm{a}}=0.56(3) \mathrm{eV}$ which is, within the error given, identical with that probed by $\operatorname{SLR}_{\rho}$ NMR (see above), the rates $1 / \tau^{\prime \prime}$ dominating echo decay at low temperatures point to an activation energy of only $0.09(1) \mathrm{eV}$. Since an almost temperature independent decay rate would be expected to account for spin-diffusion effects, as mentioned above, one might propose to identify the rate $1 / \tau^{\prime \prime}$ with a (quadrupolar or dipolar) SLR rate. Although such a rate is expected to be larger than the independently measured rate $1 / T_{1}$, the temperature dependencies of these SLR NMR rates should be comparable. Here, however, $1 / T_{1}$ (as well as $1 / T_{1 \rho}$ ) reveals a much weaker temperature dependence (see above). Thus, $1 / \tau^{\prime \prime}$ seems to contain additional information on the Li dynamics present at low temperatures which are masked in the case of $1 / T_{1}$ (and $1 / T_{1 \rho}$ ). In view of the fact that the associated activation energy is rather low (less than $0.1 \mathrm{eV}$ ), (weakly activated) localized (or caged) motions rather than long-range transport might be responsible for the process characterized by $1 / \tau^{\prime \prime}$. Interestingly, the activation energy probed is very similar to that of the strictly localized two-site jump processes in crystalline spodumene $\left(\mathrm{LiAlSi}_{2} \mathrm{O}_{6}\right)$ [44]. Further SAE NMR measurements carried out at different resonance frequencies might help to clarify the origin of the low-temperature echo decay.

\subsubsection{Impedance spectroscopy}

Long-range, i.e., end-to-end ion transport can be probed by conductivity measurements. Typical impedance spectra of the gas-phase synthesized amorphous oxide are shown in Fig. 5a where the real part, $\sigma$, of the complex conductivity is shown as a function of frequency. Typically, the spectra consist of three regimes. At low frequencies polarisation effects, due to blocking electrodes applied, lead to a decrease of $\sigma$, while at high frequencies a dispersive regime is observed. At intermediate frequencies distinct frequency-independent plateaus show up being characteristic of successful ion hops in the oxide studied.

The corresponding conductivity values, depicted in Fig. 5b, were read out at the saddle point of the impedance spectra. In a good approximation they can be identified with the dc-conductivities $\sigma_{\mathrm{dc}}$. Let us remark that almost identical values were obtained from an analysis of the corresponding Cole-Cole plots which can be constructed when the (negative) imaginary part of the complex impedance, $Z^{\prime \prime}$, is plotted $v s$ the real part, $Z^{\prime}$. The intersection of the semicircle with the real axis is given by $1 / \sigma_{\mathrm{dc}}$. As can be seen in Fig. $5 \mathrm{~b}, \sigma_{\mathrm{dc}} T$ clearly follows Arrhenius behaviour according to

$$
\sigma_{\mathrm{dc}} T=\sigma_{0} \exp \left(-E_{\mathrm{a}, \sigma} / k_{\mathrm{B}} T\right) .
$$

The fit shown (see the solid line in Fig. 5b) yields an activation energy $E_{\mathrm{a}, \sigma}$ of $0.80(1) \mathrm{eV}$. Clearly, this value is much larger than those probed by $\mathrm{SLR}_{\rho}$ and SAE NMR. Note that $\mathrm{SLR}_{\rho}$ rates have been measured at frequencies comparable to those 

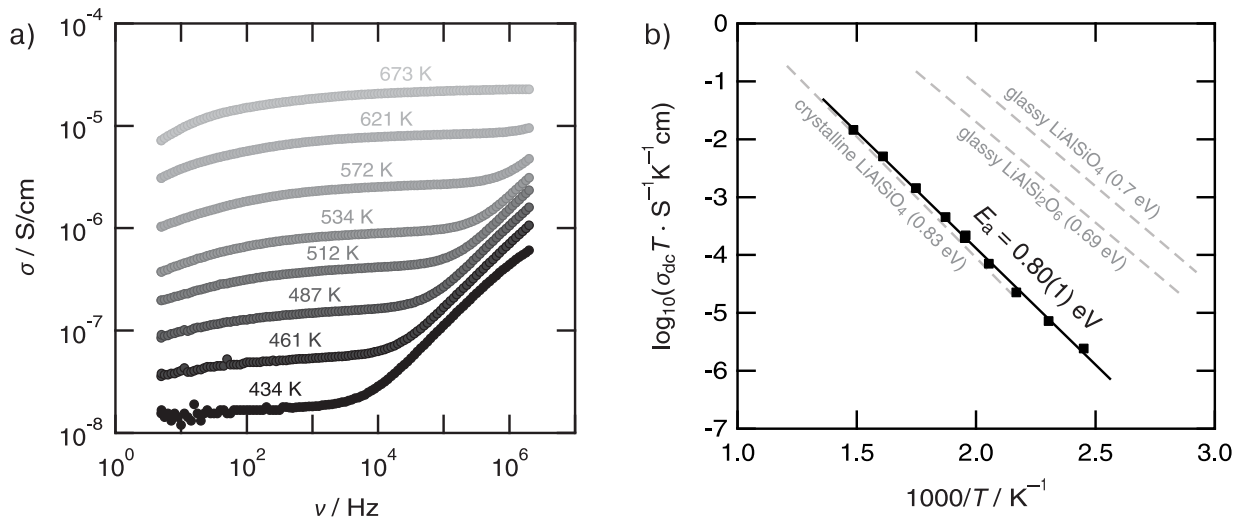

Fig. 5. (a) Impedance spectra (normalized to the sample geometry) recorded at the temperatures indicated. The spectra are composed of a "dc-plateau" and a dispersive regime showing up at higher frequencies. At lower frequencies, $\sigma$ decreases due to ion blocking polarisation effects at the electrodes. (b) The temperature dependence of $\sigma_{\mathrm{dc}} T$ shows Arrhenius behaviour with an activation energy $E_{\mathrm{a}, \sigma}=0.8 \mathrm{eV}$. For comparison, the respective Arrhenius lines of glassy and crystalline eucryptite $\left(\mathrm{LiAlSiO}_{4}\right)$ as well as glassy spodumene $\left(\mathrm{LiAlSi}_{2} \mathrm{O}_{6}\right)$ are also indicated (data were taken from Refs. [71,72]).

where the conductivity values have been determined. In the case of SLR NMR one might argue that the rates $1 / T_{1}$ are influenced by correlation effects caused by Coulomb interactions and/or structural disorder [68-70]. Such effects are known to lead to a reduced slope of the rate peak in the limit $\omega_{0} \tau \gg 1$. This behaviour is accompanied with a sub-quadratic frequency dependence of $1 / T_{1}$ on the low- $T$ side of the peak $1 / T_{1}(1 / T)$. However, since no discrepancy between the activation energies found by $\mathrm{SLR}_{\rho} \mathrm{NMR}_{\text {on }}$ the one hand and SAE NMR on the other hand is found, correlation effects seem to play a minor role in affecting SLR NMR rates measured in the low- $T$ limit here. Instead, ion blocking regions of the particle-assembled structure (see Fig. 1b) might contribute to the dc-conductivities probed. In contrast, due to the much lower number density of charge carriers in such near-surface regions, NMR data, even when sensitive to longrange motions as in the case of the SAE technique, is mainly sensitive to Li dynamics taking place in the bulk, i.e., in the interior of the particles.

For comparison, when results from bulk ion conductivities are compared with SAE NMR data, quite often good agreement is found [25]. As an example, this similarity has been shown to be valid for polycrystalline garnet-type $\mathrm{Li}_{7} \mathrm{Zr}_{2} \mathrm{La}_{3} \mathrm{O}_{12}[13,47]$ investigated quite recently by some of us.

Finally, in Fig. 5b the relatively low Li ion diffusivity and ion conductivity, respectively, found here is compared with results on crystalline and glassy eucryptite $\mathrm{LiAlSiO}_{4}$ [71] as well as glassy spodumene $\mathrm{LiAlSi}_{2} \mathrm{O}_{6}$ [72] (see also Refs. [73,74]). Interestingly, although the sample studied turns out to be X-ray amorphous, ion conductivities probed resemble that of crystalline $\mathrm{LiAlSiO}_{4}$ rather than that of the glassy counterpart. Once again this might simply be explained by a large influence of ion blocking regions between the globular particles. As indicated by the impedance spectrum recorded at $434 \mathrm{~K}$, a second frequency-independent plateau might show up at larger frequencies reflecting bulk data. Regarding the activation energies deduced from 
$\mathrm{SLR}_{\rho} \mathrm{NMR}$ and SAE NMR, the value of $0.56 \mathrm{eV}$ found for the non-stoichiometric amorphous oxide studied here is reasonably consistent with the trend shown in Fig. 5b.

\subsection{Conclusion and outlook}

Li dynamics in a gas-phase synthesized non-stoichiometric Li-Al-Si-oxide was complementarily investigated by NMR and impedance spectroscopy. The activation energy deduced from ${ }^{7} \mathrm{Li}$ NMR SLR rates probed in the rotating frame of reference $(0.56 \mathrm{eV})$ is in very good agreement with that probed by mixing-time dependent ${ }^{7} \mathrm{Li} \mathrm{SAE} N \mathrm{NR}$ being sensitive to long-range Li motions. Thus, the two methods do obviously probe the same diffusion process in the metastable oxide. Together with results from NMR line shape measurement, the findings reveal moderate Li diffusivity in the structurally disordered oxide investigated. The analysis of variable-temperature impedance spectra point to a Li transport process being characterized by an even higher activation energy of approximately $0.8 \mathrm{eV}$.

Interestingly, the low temperature SAE NMR decay rates indicate weakly activated localized Li motions characterized by $0.09 \mathrm{eV}$. Further frequency-dependent SAE NMR measurements as well as the acquisition of low- $T$ impedance spectra might help characterize this dynamic process in more detail.

\section{Acknowledgement}

We thank P. Bottke and A. Kuhn for valuable discussions. Financial support by the Deutsche Forschungsgemeinschaft (DFG) within the Research Unit 1277 "Mobilität von Li-Ionen in Festkörpern" (molife) is highly acknowledged.

\section{References}

1. H. Mehrer, Diffusion in Solids: Fundamentals, Methods, Materials, Diffusion-Controlled Processes, Springer, Berlin (2007).

2. P. Heitjans and J. Kärger (Eds.), Diffusion in Condensed Matter: Methods, Materials, Models, Springer, Berlin (2005).

3. A. V. Chadwick, Diffus. Fundam. 2 (2005) 44.

4. P. Heitjans, S. Indris, and M. Wilkening, Diffus. Fundam. 2 (2005) 45.

5. V. W. J. Verhoeven, I. M. de Schepper, G. Nachtegaal, A. P. M. Kentgens, E. M. Kelder, J. Schoonman, and F. M. Mulder, Phys. Rev. Lett. 86 (2001) 4314.

6. J. Cabana, N. Dupré, G. 1. Rousse, C. P. Grey, and M. R. Palacín, Solid State Ionics 176 (2005) 2205.

7. M. Wilkening, W. Küchler, and P. Heitjans, Phys. Rev. Lett. 97 (2006) 065901.

8. L. S. Cahill, R. P. Chapman, J. F. Britten, and G. R. Goward, J. Phys. Chem. B 110 (2006) 7171.

9. L. van Wüllen, T. Echelmeyer, H.-W. Meyer, and D. Wilmer, Phys. Chem. Chem. Phys. 9 (2007) 3298.

10. M. Wilkening and P. Heitjans, Phys. Rev. B 77 (2008) 024311.

11. B. Koch and M. Vogel, Solid State Nucl. Mag. 34 (2008) 37.

12. J. Cabana, J. Shirakawa, G. Chen, T. J. Richardson, and C. P. Grey, Chem. Mater. 22 (2010) 1249.

13. A. Kuhn, S. Narayanan, L. Spencer, G. Goward, V. Thangadurai, and M. Wilkening, Phys. Rev. B 83 (2011) 094302. 
14. H. Hain, M. Scheuermann, R. Heinzmann, L. Wünsche, H. Hahn, and S. Indris, Solid State Nucl. Mag. 42 (2012) 9.

15. A. Kuhn, M. Kunze, P. Sreeraj, H. D. Wiemhöfer, V. Thangadurai, M. Wilkening, and P. Heitjans, Solid State Nucl. Mag. 42 (2012) 2.

16. M. Armand and J.-M. Tarascon, Nature 451 (2008) 652.

17. P. Knauth, Solid State Ionics 180 (2009) 911.

18. H. Buschmann, J. Dölle, S. Berendts, A. Kuhn, P. Bottke, M. Wilkening, P. Heitjans, A. Senyshyn, H. Ehrenberg, A. Lotnyk, V. Duppel, L. Kienle, and J. Janek, Phys. Chem. Chem. Phys. 13 (2011) 19378.

19. P. Heitjans, M. Masoud, A. Feldhoff, and M. Wilkening, Faraday Discuss. 134 (2007) 67.

20. M. Wilkening, V. Epp, A. Feldhoff, and P. Heitjans, J. Phys. Chem. C 112 (2008) 9291.

21. M. J. Pooley and A. V. Chadwick, Radiat. Eff. Defects Solids. 158 (2003) 209.

22. S. Faske, H. Eckert, and M. Vogel, Phys. Rev. B 77 (2008) 104301.

23. M. Wilkening, A. Kuhn, and P. Heitjans, Phys. Rev. B 78 (2008) 054303.

24. P. Heitjans, A. Schirmer, and S. Indris, NMR and $\beta$-NMR studies of diffusion in interfacedominated and disordered solids, in: Diffusion in Condensed Matter: Methods, Materials, Models, P. Heitjans, J. Kärger (Eds.), Springer, Berlin (2005), pp. 367-415.

25. M. Wilkening and P. Heitjans, Chem. Phys. Chem. 13 (2012) 53.

26. M. Wilkening and P. Heitjans, Solid State Ionics 177 (2006) 3031.

27. M. Wilkening and P. Heitjans, Defect Diffus. Forum 237-240 (2005) 1182.

28. R. Böhmer, T. Jörg, F. Qi, and A. Titze, Chem. Phys. Lett. 316 (2000) 419.

29. F. Qi, T. Jörg, and R. Böhmer, Solid State Nucl. Mag. 22 (2002) 484.

30. F. Qi, G. Diezemann, H. Böhm, J. Lambert, and R. Böhmer, J. Magn. Reson. 169 (2004) 225.

31. R. Böhmer and F. Qi, Solid State Nucl. Mag. 31 (2007) 28.

32. S. Faske, B. Koch, S. Murawski, R. Küchler, R. Böhmer, J. Melchior, and M. Vogel, Phys. Rev. B 84 (2011) 024202.

33. T. Giesenberg, S. Hein, M. Binnewies, and G. Kickelbick, Angew. Chem. Int. Edit. 43 (2004) 5697.

34. C. Brünig, Ph.D. thesis, Leibniz University Hannover (2010).

35. E. Fukushima and S. B. W. Roeder, Experimental Pulse NMR: A Nuts and Bolts Approach, Addison-Wesley Pub. Co., Reading, Mass. (1981).

36. D. Ailion and C. P. Slichter, Phys. Rev. Lett. 12 (1964) 16.

37. C. P. Slichter and D. C. Ailion, Phys. Rev. 135 (1964) A1099.

38. D. C. Ailion and C. P. Slichter, Phys. Rev. 137 (1965) A235.

39. D. C. Look and I. J. Lowe, J. Chem. Phys. 44 (1966) 2995.

40. T. J. Rowland and F. Y. Fradin, Phys. Rev. 182 (1969) 760.

41. D. Wolf, Phys. Rev. B 10 (1974) 2724.

42. J. Jeener and P. Broekaert, Phys. Rev. 157 (1967) 232.

43. R. Böhmer, J. Magn. Reson. 147 (2000) 78.

44. F. Qi, C. Rier, R. Böhmer, W. Franke, and P. Heitjans, Phys. Rev. B 72 (2005) 104301.

45. X.-P. Tang and Y. Wu, J. Magn. Reson. 133 (1998) 155.

46. R. Böhmer, K. Jeffrey, and M. Vogel, Prog. Nucl. Magn. Res. Spectrosc. 50 (2007) 87.

47. A. Kuhn, V. Epp, G. Schmidt, S. Narayanan, V. Thangadurai, and M. Wilkening, J. Phys.Condens. Matter 24 (2012) 035901.

48. M. Wilkening, J. Heine, C. Lyness, A. R. Armstrong, and P. G. Bruce, Phys. Rev. B 80 (2009) 064302.

49. H. W. Spiess, J. Chem. Phys. 72 (1980) 6755.

50. M. Lausch and H. W. Spiess, J. Magn. Reson. (1969-1992) 54 (1983) 466.

51. F. Fujara, S. Wefing, and H. W. Spiess, J. Chem. Phys. 84 (1986) 4579.

52. J. S. Waugh and E. I. Fedin, Sov. Phys. Solid State 4 (1963) 1633.

53. D. Tse and S. R. Hartmann, Phys. Rev. Lett. 21 (1968) 511.

54. H.-J. Stöckmann and P. Heitjans, J. Non-Cryst. Solids 66 (1984) 501.

55. W.-K. Lee, J. F. Liu, and A. S. Nowick, Phys. Rev. Lett. 67 (1991) 1559.

56. A. S. Nowick, B. S. Lim, and A. V. Vaysleyb, J. Non-Cryst. Solids 172-174 (1994) 1243.

57. H. Jain and S. Krishnaswami, Solid State Ionics 105 (1998) 129. 
58. A. K. Rizos, J. Alifragis, K. L. Ngai, and P. Heitjans, J. Chem. Phys. 114 (2001) 931.

59. K. Funke, R. D. Banhatti, D. M. Laughman, L. G. Badr, M. Mutke, A. Šantić, W. Wrobel, E. M. Fellberg, and C. Biermann, Z. Phys. Chem. 224 (2010) 1891.

60. K. L. Ngai, U. Strom, and O. Kanert, Phys. Chem. Glasses 33 (1992) 109.

61. O. Kanert, R. Küchler, J. Dieckhöfer, X. Lu, and H. Jain, Phys. Rev. B 49 (1994) 629.

62. O. Kanert, R. Küchler, P. C. Soares, and H. Jain, J. Non-Cryst. Solids 307 (2002) 1031.

63. J. Van Kranendonk, Physica 20 (1954) 781.

64. R. L. Mieher, Phys. Rev. 125 (1962) 1537.

65. N. Bloembergen, E. M. Purcell, and R. V. Pound, Phys. Rev. 73 (1948) 679.

66. A. Kuhn, diploma thesis, Leibniz University Hannover (2008).

67. P. Heitjans, E. Tobschall, and M. Wilkening, Eur. Phys. J.-Spec. Top. 161 (2008) 97.

68. M. Meyer, P. Maass, and A. Bunde, Phys. Rev. Lett. 71 (1993) 573.

69. A. Bunde, W. Dieterich, P. Maass, and M. Meyer, Ionic transport in disordered materials, in: Diffusion in Condensed Matter: Methods, Materials, Models, P. Heitjans, J. Kärger (Eds.), Springer, Berlin (2005), pp. 813-856.

70. K. Funke, Prog. Solid State Chem. 22 (1993) 111.

71. P. Bottke, diploma thesis, Leibniz University Hannover (2010).

72. A. Kuhn, M. Wilkening, and P. Heitjans, Solid State Ionics 180 (2009) 302.

73. B. Munro, M. Schrader, and P. Heitjans, Ber. Bunsen. Phys. Chem. 96 (1992) 1718.

74. A.-M. Welsch, H. Behrens, I. Horn, S. Roß, and P. Heitjans, J. Phys. Chem. A 116 (2012) 309. 